\title{
Pharmacokinetic aspects of rectal formulations of Temazepam
}

- L.M. Hanff and W.J. Rutten

\section{Introduction}

Temazepam is one of the most-used hypnotics world wide. Only oral formulations of temazepam are commercially available: a powder-filled capsule and a liquid-tilled capsule, the latter showing a more rapid absorption than the former [1 12$]$.

In clinical practice, the liquid-filled temazepam capsules are occasionally administered rectally in patients for hypnotic purposes, when oral intake is not possible. No data, however, are available on the rectal use of these capsules.

Several authors have descibed rectal administration of benzodia zepines such as diazepam, flunitrazepam and nitrazepam (for a review see [3]). Fucella [4] published in 1972 a study in four volunteers on the bioavailability of temazepam after rectal administration in a suppository, in comparison with the oral use of temazepam in a capsule and a solution. The bioavailability of temazepam from suppositories ranged between $57 \%-90 \%$ of the bioavailability of temazepam from the capsules. However, the composition of neither the suppository base, the capsule nor the oral solution was mentioned.

The aim of this study was to develop a suitable rectal formulation for temazepam by determination of pharmacokinetic data in volunteers after rectal administration of three formulations of temazepam: a suppository, a micro-enema and a commercially available liquid-filled capsule. Oral use of the liquid-filled capsule was used as a reference. An in vitro study with temazepam in three different suppository bases was undertaken and release parameters were determined in order to select a suitable suppository base for in vivo testing. The release profile of the liquid-filled capsule was determined in vitro to allow comparison of in vivo-in vitro data.

\section{Methods}

In vitro study

In vitro release of temazepam from three different suppositories and the liquid-filled capsule (Normison®, Wyeth Laboratoria, Hoofddorp, the Netherlands) was studied using the paddle apparatus, modified by Cox [3]. Demineralised water $(1000 \mathrm{ml})$ was used as dissolution medium at a temperature of $37 \pm 0.5^{\circ} \mathrm{C}$. Stirring rate was adjusted to $60 \mathrm{rpm}$.

Temazepam suppositories $(2.3 \mathrm{ml})$ were extemporarily prepared with the following bases: polyethylene glycol (PEG) 1540, PEG 1540:4000 (2:1) and fatty base (Witepsol H15). All compounds were obtained from Bufa Chemicals, Uitgeest, the Netherlands. The liquid-filled capsules contained a solution of temazepam in polyethylene glycol and glycerol in a soft gela. tin capsule. All formulations contained $10 \mathrm{mg}$ of temazepam and complied with the $95-105 \%$ content range and the test on uniformity of content on singledose preparations, described in Eur.Ph. 2nd ed. [5].
L.M. Hanff, W.J. Rutten. Pharmacokinetic aspects of rectal formulations of Temazepam

Pharm World Sci 1996; 18(3): 114-119.

01996 Kluwer Academic Publishers. Printed in the Nethedands.

L.M. Hanff: Department of Pharmacy, University Hospital Rotterdam, Dr. Molewaterplein 40, 3015 CD Rotterdam, the Netherlands (correspondence), W.J. Rutten: Department of Clinical Pharmacy, 'Medisch Spectrum Twente', Ariensplein 1, Enschede, the Netherlands.

\section{Keywords}

Temazepam

Rectal administration

Suppository

Capsule

Enema

Pharmacokinetic

\section{Abstract}

An in vitro/in vivo study was carried out with different rectal formulations of temazepam. Pharmacokinetic data were determined in a cross-over study in 10 volunteers after rectal administration of $10 \mathrm{mg}$ temazepam as a polyethylene glycol based suppository (selected from in vitro data), a liquid-filled capsule and a micro-enema respectively, using oral administration of a liquid-filled capsule as a reference. Serum levels of temazepam indicate an instantaneous and complete release from the micro-enema $\left(F_{r e t}=0.94 \pm 0.21\right.$

$C_{\max } 205 \pm 36.9 \mu \mathrm{g} / \mathrm{l}, \mathrm{t}_{\max } 0.49 \pm 0.31$ hour) and a slower but complete release of temazepam from the suppository $\left(F_{\text {ret }}=1.07 \pm 0.25, C_{\text {max }} 202 \pm 41.3 \mu \mathrm{g} / \mathrm{l}, t_{\mathrm{max}} 1.48 \pm 0.41\right.$ hour). A high interindividual variation in absorption profiles was observed after rectal administration of the liquid-filled capsule $\left(F_{\text {re }} 0.72 \pm 0.36, C_{\text {a }} 182 \pm 122 \mu g / 1, t_{\text {max }} 4.08\right.$ \pm 4.28 hour), which makes it less suitable for rectal use. The micro-enema and suppository appear to be useful as rectal formulations for temazepam. 
immersed $2 \mathrm{~cm}$ below the dissolution medium at $\mathrm{t}=$ 0 . Samples of the dissolution medium were taken at $\mathrm{t}=0 ; 2.5 ; 5 ; 7.5 ; 10 ; 15 ; 20 ; 25 ; 30 \mathrm{~min}$.

Samples were analysed by measuring absorbance at $230 \mathrm{~nm}$ (Hitachi spectrophotometer 150-20 in combination with Hitachi Data processor 150-20, Merck, Nieuwekerk a/d IJssel, the Netherlands). Standards of temazepam $(12.5,6.25,3.125$ and $1.562 \mathrm{mg} / \mathrm{l}$ temazepam in water) were prepared by dilution of a stock solution of temazepam $250 \mathrm{mg} / \mathrm{l}$ in methanol:water (150:350). Lower assay limit was 0.5 $\mathrm{mg} / \mathrm{l}$, correlation coefficient of the calibration curves was $0.9998(\mathrm{~N}=6)$.

For each formulation, release profiles were determined ( $N=7$ for the suppositories, $n=8$ for the liquidfilled capsule). Release parameters were calculated with the software program WEIFIT [6], according to the formula $F=F u .\left(1-e^{-[(t-T O / T d] \beta}\right)$, in which

$\mathrm{F}=$ amount released at time $\mathrm{t}(\mathrm{mg})$

$\mathrm{Fu}=$ amount released after infinite time $(\mathrm{mg})$

$\mathrm{TO}=$ lag time $(\mathrm{min})$

$\mathrm{Td}=$ time to release $63.2 \%$ of the total dose $(\mathrm{min})$

$B=$ shape parameter

$\mathrm{t}=$ time $(\mathrm{min})$

\section{Statistical analysis}

Differences in means of $\mathrm{Fu}, \mathrm{TO}$ and $\mathrm{Td}$ of the formulations were analysed for statistical significance by Student's $t$-test for unpaired data. $P$ values $<0.05$ were considered as statistically significant.

\section{In vivo study}

\section{Study population}

The study was conducted at the hospital 'Medisch Spectrum Twente' in Enschede, the Netherlands.

Ten healthy, male volunteers between 21 and 52 years participated in the study on a informed consent base. Excluded were subjects with known intolerance to benzodiazepines.

The study was approved by the local Institutional Review Board.

\section{Study design}

A cross-over design was used in the study in order to minimize interindividual differences.

The suppository, micro-enema and liquid-filled capsule were self-administered rectally by each volunteer in randomized order. Oral administration of the liquid-filled capsule took place after overnight fasting, after which volunteers continued fasting for another 3 hours.

A wash-out period of one week was maintained between the administration of the different formulations. During the first hour after rectal administration the volunteers stayed in lying position, in order to minimize variability in rectal absorption.

After completion of the study, volunteers were asked for their preference in rectal formulations, with regard to handling and comfort.

\section{Dosage forms}

Based on the data of the in vitro study (see Results), temazepam suppositories $(2.3 \mathrm{ml})$ were prepared in a polyethylene glycol 1540 base.

The micro-enema contained temazepam in a $2 \mathrm{ml}$ solution of glycofurol:ethanol $96 \%$ :water (5:1:4), sim- ilar to the composition of diazepam micro-enemas [3]. Methylhydroxybenzoate $(0.15 \%)$ was added as a preservant. The liquid-filled capsule (Normison®) was used both orally and rectally.

All preparations contained $10 \mathrm{mg}$ of temazepam.

\section{Sample collection}

A catheter (Insyte-W $1.3 \times 32 \mathrm{~mm}$, Vialon $®$, Becton Dickinson, USA) was inserted in a forearm vein to facilitate venous blood sampling. Samples were obtained prior to temazepam administration and at $0.08^{\mathrm{a}}, 0.16^{\mathrm{a}}, 0.25,0.5,1.0,1.5^{\mathrm{b}}, 2.0,3.0^{\mathrm{b}}, 4.0,6.0$, 8.0, 10.0, 24.0 hours after temazepam administration ( ${ }^{\mathrm{a} m i c r o-e n e m a, ~ b l i q u i d-f i l l e d ~ c a p s u l e ~ r e c t a l l y) . ~ E a c h ~}$ time, $5 \mathrm{ml}$ blood were collected in a glass tube (Venoject $B$, Terumo, Leuven, Belgium) and centrifuged, after clotting. The serum samples were stored at $-20{ }^{\circ} \mathrm{C}$ in polypropylene tubes (Sarsted, Numbrecht, Germany) until analysis.

\section{Assay}

Serum levels of temazepam and oxazepam, a very minor metabolite, were determined using a reversedphase high performance liquid chromatography system (Perkin-Elmer Series 10 Liquid Chromatography), provided with an autosampler (PerkinElmer ISS-101, Amsterdam, the Netherlands) [7, modified].

One $\mathrm{ml}$ of serum was mixed with $100 \mu$ internal standard (camazepam $2 \mathrm{mg} / \mathrm{l}$ in methanol:water [150:350]) and $0.4 \mathrm{ml}$ sodium borate buffer $\mathrm{pH} 9.0$ $\left(\mathrm{Na}_{2} \mathrm{~B}_{4} \mathrm{O}_{7} \bullet 10 \mathrm{H}_{2} \mathrm{O} 0.05 \mathrm{M}: \mathrm{HCl} 0.1 \mathrm{~N}[85: 15]\right) .5 \mathrm{ml}$ of dichloromethane were added as extraction medium. The mixture was shaken for $15 \mathrm{~min}$, and centrifuged for $5 \mathrm{~min}$ at 18009 . The organic phase was evaporated under a flow of nitrogen. After reconstitution in $100 \mu \mathrm{l}$ of mobile phase (methanol:acetonitril: potassiumdihydrogenphosphate $0.05 \mathrm{M}$ [440:165:570]), $60 \mu$ were injected on a LiChrosphere 100RP-18 (5 $\mu \mathrm{m}$ ) column (Merck 50943). At a flow rate of 1.5 $\mathrm{ml} / \mathrm{min}$, retention times of oxazepam, temazepam and camazepam were respectively $4.3,5.5$ and 11.5 min. The absorbance was monitored at wavelength $230 \mathrm{~nm}$, using a Perkin-Flmer LC-75 spectrophotometer (Amsterdam, the Netherlands). All chemicals were obtained from Merck, Darmstadt, Germany.

Two sets of standards (serum, spiked with 10, 25, $50,100,200,400 \mu \mathrm{g} / \mathrm{l}$ of oxazepam and temazepam) and two control sera (KKGT: Association for External Quality Assessment in TDM \& Toxicology, the Hague, the Netherlands) were run with each batch of samples. Using the standard curves, serum concentrations were calculated with Turbochrom III (Perkin-Elmer) software system. The assay was validated for the serum level-range between $10 \mu \mathrm{g} / \mathrm{l}$ and $400 \mu \mathrm{g} / \mathrm{l}$ for oxazepam and temazepam [8].

Recovery of oxazepam and temazepam was $92.4 \%$ and $96.7 \%$ respectively. The lower assay limit was 10 $\mu \mathrm{g} / \mathrm{l}$, the limit of detection was $2 \mu \mathrm{g} / \mathrm{l}$ for both oxazepam and temazepam. The standard deviation $(y)$ of the assay, in relation to the serum level $(x)$, was described by the following polynome $y=2.01+$ $5.27 * 10^{-3} x+7.27^{*} 10^{-5} x^{2}-6.38^{*} 10^{-8} x^{3} \quad(n=6)$ for temazepam and $y=-0.09+9.99^{*} 10^{-2} x-5.71 * 10^{-4} x^{2}$ $+1.07^{\star} 10^{-6} x^{3}(n=6)$ for oxazepam. 
temazepam released [\%]

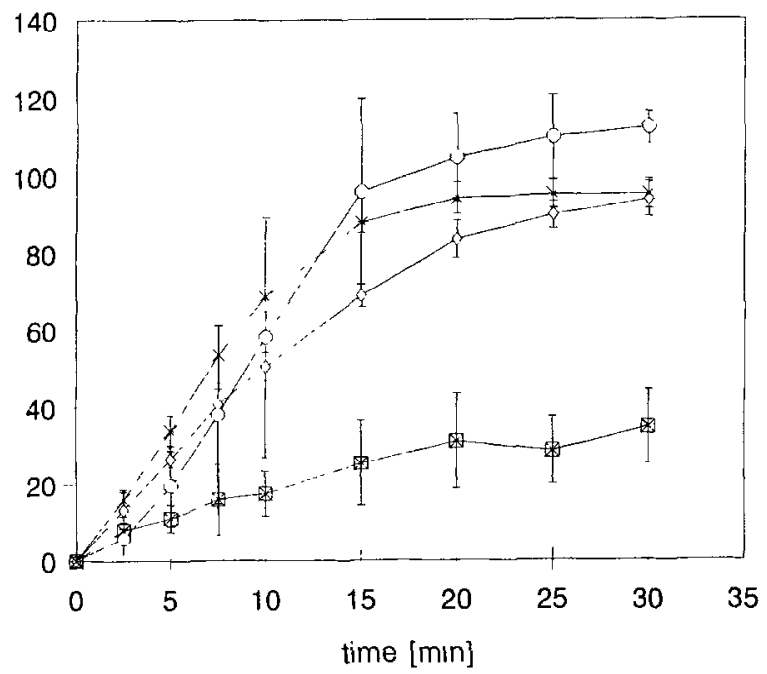

Figure 1

In vitro release profiles of rectal formulations of $10 \mathrm{mg}$ Temazepam.

\section{Pharmacokinetic and statistical analysis}

Pharmacokinetic parameters were calculated with the software program KINFIT (Mediware ${ }^{\circledR}$, Groningen, the Netherlands). Non-linear curve fitting based on a two-compartment model provided an estimation of peak concentration $\left(C_{\max }[\mu \mathrm{g} / \mathrm{l}]\right)$, time to peak concentration $t_{\max }$ [hour], lag time [hour], absorption rate constant $\mathrm{ka}$ [1/hour], clearance $\mathrm{Cl}[1 /$ hour], elimination half-life $t^{1} / 2 \mathrm{el}$. [hour] and area under the curve (AUC [hour, $\mu \mathrm{g} / \mathrm{l}], \mathrm{t}=0-24$ hour) calculated by the trapezoidal rule. The relative bioavailability $\left(F_{\text {rel }}\right)$ was calculated by dividing the AUC of the rectal formulation by the AUC after oral intake of the liquid-filled capsule. The cumulative input of the different rectal formulations was calculated by numerical deconvolution, using the software program KINBES. The mean differences of the interindividual parameters using rectal formulations were tested for statistical significant differences with the results after oral administration, using the Student's $t$-test for paired data ( $P<$ 0.05 ).

\section{Results}

In vitro study

The release profiles are shown in Figure 1. Release parameters, calculated by WEIFIT, are summarized in
Table 1. Despite complete melting of the suppositories within 10 minutes, release of temazepam from the fatty base was incomplete and inconsistent and release parameters could not be calculated by curvefitting. The suppositories with polyethylene glycol bases showed a reproducible and complete release of temazepam within 30 minutes. No significant differences in Fu were found between the two polyethylene glycol bases. However, Td of the polyethylene glycol 1540 base was significantly lower than $\mathrm{Td}$ of the polyethylene glycol 1540:4000 base, indicating a faster release of temazepam from polyethylene glycol 1540. The liquid-filled capsules showed an inconsistent release with a high variation in lag-time and $\mathrm{Td}$ between the capsules tested. However, within 20 minutes the release process was completed.

\section{In vivo study}

All volunteers completed the study. No side-effects were experienced, except for a slight drowsiness in some volunteers.

The serum concentration-time profiles and the cumulative input of the different rectal formulations and the oral formulation are shown in Figure 2a-d. The mean pharmacokinetic parameters and their corresponding standard deviations are summarized in Table 2.

The AUC of both micro-cnema and suppository did not differ significantly from the AUC of the oral capsule, indicating an equivalent bioavailability. However, the AUC after rectal administration of the capsule, was significant lower $(P<0.05)$ than the AUC after oral administration. After administration of the micro-enema absorption started almost immediately. In comparison to the micro-enema and the oral capule, the suppository showed a slower absorption process (ka $1.5 \pm 1.3$ hour $^{-1}$ ) and a significant higher $t_{\max }(1.5 \pm 0.4$ hour $)$.

The liquid-filled capsule showed an highly erratic absorption-profile after rectal administration. Only minor absorption occurred in five of the ten volunteers, with hardly any absorption in two volunteers. The other volunteers showed absorption-profiles comparable with oral intake. Serum-concentrations of oxazepam were below quantification levels in all samples. The observed pharmacokinetic parameters after oral administration of the liquid-filled capsule: $\mathrm{Cl} 81 \pm$ $25 \mathrm{ml} / \mathrm{kg} /$ hour and $\mathrm{t}^{1} / 2$ el. $9.3 \pm 5.4$ hour (Table $2, \mathrm{Cl}$ corrected for body-weight of the volunteers) were similar to data reported in literature $(\mathrm{Cl} 60-120$ $\mathrm{ml} / \mathrm{kg} /$ hour, $\mathrm{t}^{1 / 2}$ el. 6-11 hour) [9].

Table 1 in vitro release parameters ( \pm standard deviation) of $10 \mathrm{mg}$ temazepam in different rectal formulations.

\begin{tabular}{lllll}
\hline & Liquid-filled capsule & PEC 1540 & PEG 1540:4000 & Fatty base \\
\hline Fu & $12.1 \pm 1.92$ & $9.6 \pm 0.40^{*}$ & $10.7 \pm 1.90^{*}$ & N.F. $^{+}$ \\
Td & $13.1 \pm 7.82$ & $8.6 \pm 0.54^{\#}$ & $14.4 \pm 5.12^{*}$ & N.F. $^{+}$ \\
B & $2.2 \pm 0.55$ & $1.5 \pm 0.08$ & $1.1 \pm 0.12$ & $0.79 \pm 0.24$ \\
T0 & $0.3 \pm 0.62$ & $0.0 \pm 0.00$ & $0.4 \pm 0.68$ & $0.22 \pm 0.45$ \\
\hline
\end{tabular}

* Fu (PEG1540) not statistically significant different from Fu (PEG 1540/4000).

\# Td (PEG1540) significantly shorter than Td (PEC1540/4000) $(P<0.01)$.

+ not to be fitted. 
temazepam plasma-concentration $[\mu \mathrm{g} / \mathrm{l}]$

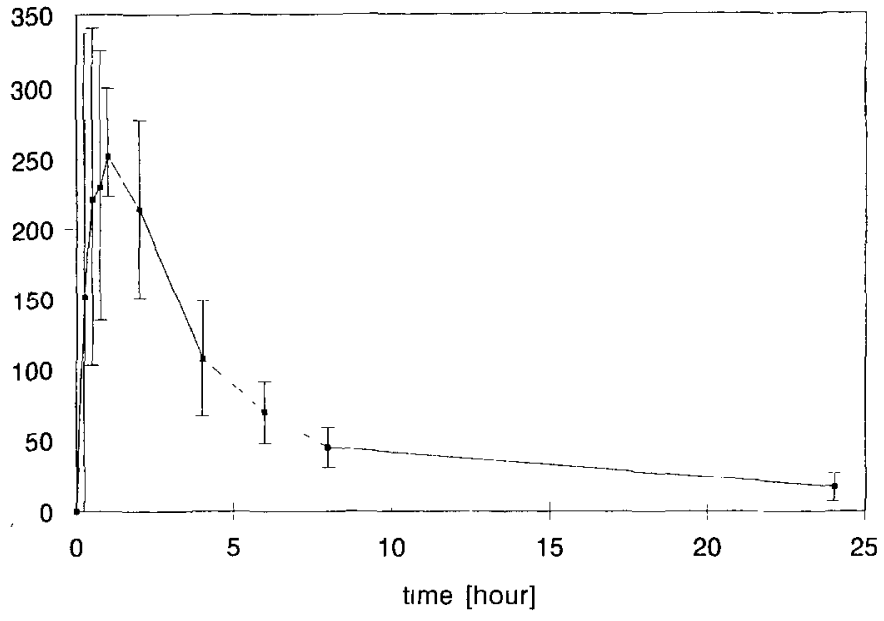

Figure 2a Serum-concentration vs. time profiles after oral administration of $10 \mathrm{mg}$ temazepam in a liquid filled capsule $(n=10)$.

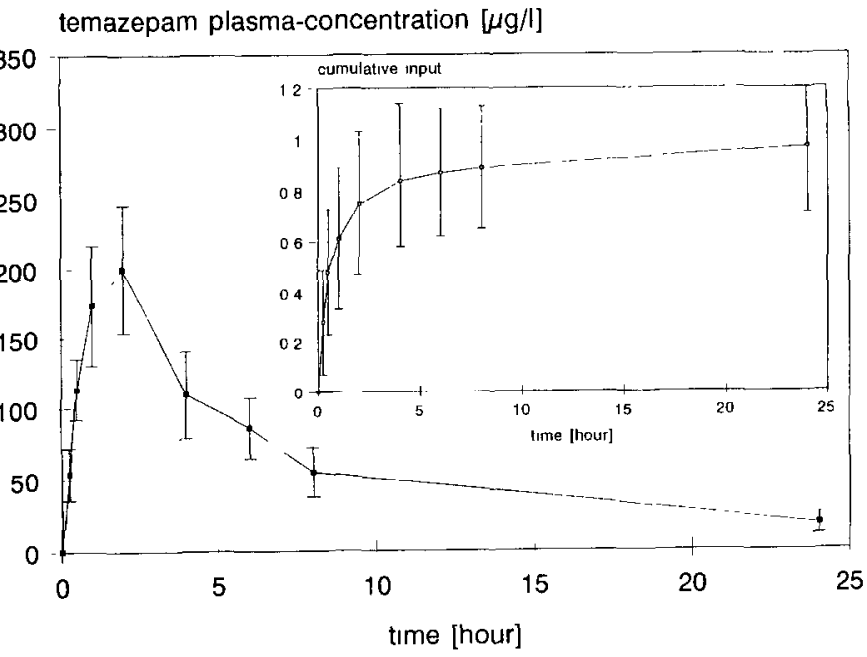

Figure 2c

Serum-concentration vs. time profiles and cumulative input (expressed in relative units, compared to oral input) after rectal administration of $10 \mathrm{mg}$ temazepam in a PEG suppository $(n=10)$

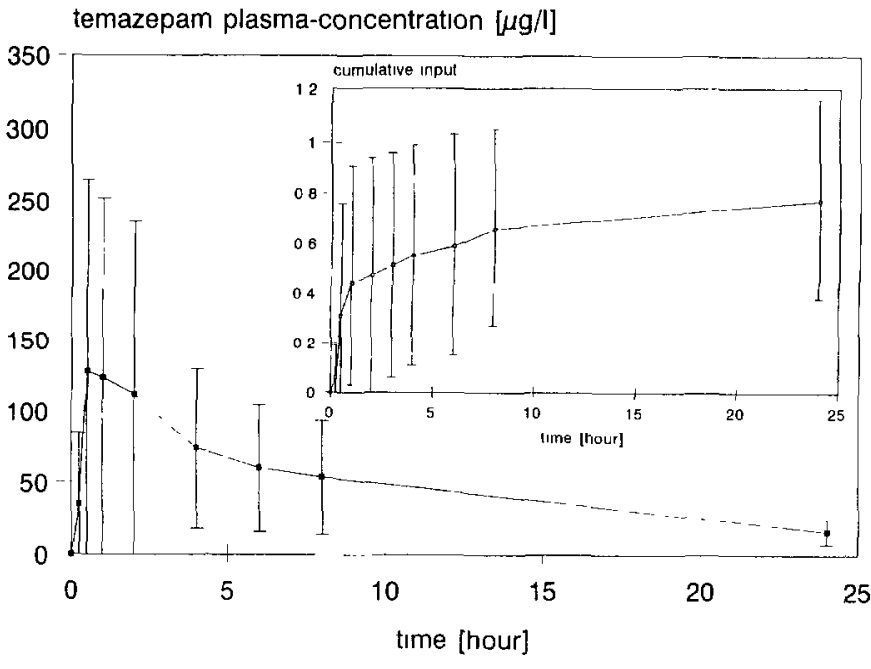

Figure 2b

Serum-concentration vs. time profiles and cumulative input (expressed in relative units, compared to oral input) after rectal administration of $10 \mathrm{mg}$ temazepam in a liquid-filled capsule $(n=10)$

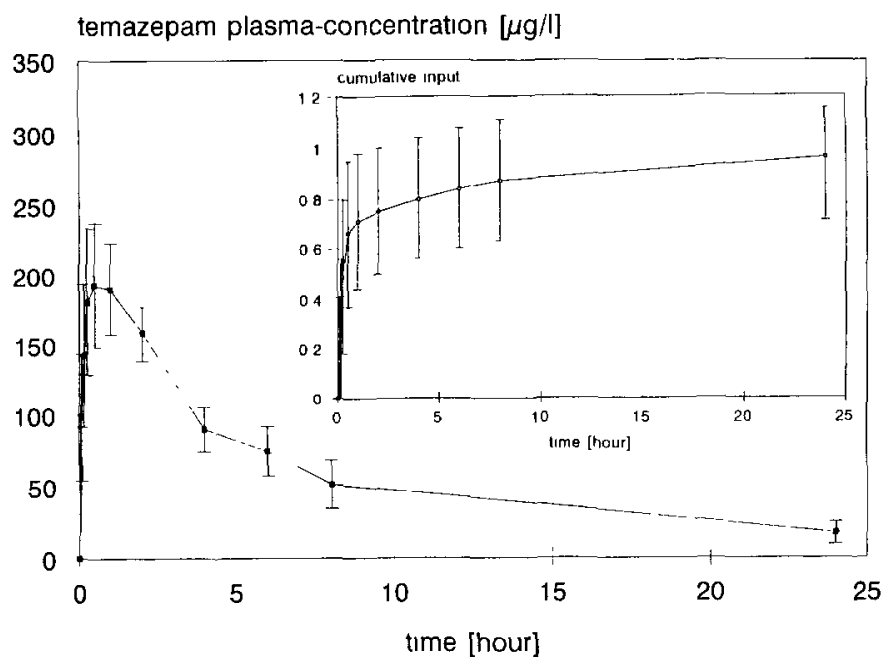

Figure 2d

Serum-concentration vs. time profiles and cumulative input (expressed in relative units, compared to oral input) after rectal administration of $10 \mathrm{mg}$ temazepam in a glycofural/ethanol/water enema $(n=10)$.

Table 2 Mean pharmacokinetic parameters ( \pm standard deviation) after oral and rectal administration of $10 \mathrm{mg}$ temazepam in different formulations to 10 volunteers

\begin{tabular}{|c|c|c|c|c|}
\hline $\begin{array}{l}\text { Pharmacokinetic } \\
\text { parameters }\end{array}$ & $\begin{array}{l}\text { Capsule } \\
\text { orally }\end{array}$ & $\begin{array}{l}\text { Capsule } \\
\text { rectally }\end{array}$ & Micro-enema & Suppository \\
\hline $\begin{array}{l}\text { AUC } C_{\mathrm{tr}} \\
\text { [hour, } \mu \mathrm{g} / \mathrm{l}]\end{array}$ & $1540 \pm 413$ & $1170 \pm 735$ & $1400 \pm 272$ & $1510 \pm 334$ \\
\hline $\mathrm{F}_{\text {rel }}$ & $1.0^{\neq}$ & $0.72 \pm 0.36$ & $0.94 \pm 0.21^{*}$ & $1.01 \pm 0.25^{*}$ \\
\hline$t_{\max }$ [hour] & $0.8 \pm 0.6$ & $4.1 \pm 4.3^{+}$ & $0.5 \pm 0.3^{*}$ & $1.5 \pm 0.4^{\#}$ \\
\hline$C_{\max }[\mu g / 1]$ & $326 \pm 76.6$ & $182 \pm 122^{+}$ & $205 \pm 36.9^{+}$ & $202 \pm 41.3^{+}$ \\
\hline lag-time [hour] & $0.2 \pm 0.2$ & $2.7 \pm 4.2^{*}$ & $0.01 \pm 0.02^{+}$ & $0.1 \pm 0.1^{*}$ \\
\hline ka [1/hour $]$ & $37.3 \pm 67.5$ & $6.9 \pm 9.8$ & $15.6 \pm 25.9$ & $1.5 \pm 1.3$ \\
\hline$t^{1} / 2$ el. [hour] & $9.3 \pm 5.4$ & $11.0 \pm 4.1^{*}$ & $7.6 \pm 5.7^{*}$ & $14.1 \pm 6.7^{\star}$ \\
\hline $\mathrm{Cl}[\mathrm{l} / \mathrm{hour}]$ & $6.4 \pm 2.0$ & $8.5 \pm 4.6^{*}$ & $7.5 \pm 2.3^{*}$ & $5.9 \pm 2.0^{*}$ \\
\hline
\end{tabular}

* By definition 1.0 .

* Not statistically significant from capsule orally.

* $p<0.05$ compared to capsule orally.

$+p<0.01$ compared to capsule orally. 
$\mathrm{Cl}$ and $\mathrm{t}^{1 / 2} \mathrm{el}$. of the rectal formulations did not differ significantly from $\mathrm{Cl}$ and $\mathrm{t}^{1} / 2 \mathrm{el}$. after oral administration, justifying the use of oral data as referencedata.

With respect to handling of the drugs, the selfadministration of the capsule was experienced as most difficult $(n=9)$ because of 'sliding problems' and the suppository as most easy $(n=9)$. With regard to comfort, 5 volunteers reported no discomfort with any of the rectal formulations. Minor local irritation was experienced with the suppositories $(n=3)$, the enema $(n=1)$ and the capsule $(n=1)$.

\section{Discussion}

\section{In vitro study}

The release data from the polyethylene glycol bases correspond with the expected optimal release of hydrophobic compounds like temazepam from a water-soluble base like PEG [3]. Comparing the PEC bases studied, the fastest release of temazepam is obtained with the PEG 1540 base, as quantified by the lowest $\mathrm{Td}$. These findings correlate with a higher melting point of a suppository base composed of a mixture of PEC 1540 and PEC 4000 compared to purely PEC 1540. Release of temazepam from a fatty base was slow, probably due to adherence of the hydrophobic compound to the base and wetting problems. Similar results have been found for the release of diazepam from a fatty base [10]. An almost instantenous release of temazepam from the liquidfilled capsules was shown due to 'bursting' of the capsule, but the period to release varied from 5 to 15 minutes after immersing the capsule. An explanation for these differences is difficult to give, however slight sticking of the capsule to the immersing device may have influenced the release process. Therefore, the results of the capsule were not included in the statistical analysis.

\section{In vivo study}

For patients who cannot take a hypnotic orally, a rectal formulation can be an easy and safe alternative to parenteral administration of a benzodiazepine. In the literature [3], rectal administration of benzodiazepines for purposes other than hypnotic use, has shown to be effective: a diazepam enema and a midazolam enema were effective as anti-epileptic and pre-medication respectively.

A optimal rectal formulation of a drug for hypnotic purposes should have the following characteristics: non-irritant, easy to prepare and to (self)-administer, no lag-time, a short $\mathrm{t}_{\max }$ and an bioavailability equivalent to oral use.

The hypnotic temazepam has the advantages of an intermediate $t^{1} / 2$ el. (6-11 hour), no active metabolites (in clinically relevant concentrations) and a worldwide experience of many years. Considering the need for a rectal hypnotic, development of a suitable rectal formulation of temazepam could mean a significant improvement in clinical practice.

In this study, none of the patients fell asleep after using either rectal or oral formulation of $10 \mathrm{mg}$ temazepam. These findings are consistent with other day-time studies with hypnotics. Since no objective drawn on hypnotic effects of either formulation, other than interpretating the pharmacokinetic data. Looking only at the $\mathrm{C}_{\max }$ of temazepam, obtained with the rectal formulations, none of the formulations appears to be in the therapeutic range described in literature $(300-800 \mu \mathrm{g} / \mathrm{l})$ [11]. However, a clear relation between serum levels and hypnotic effect has never been established for temazepam. An effective rectal dosage of temazepam should probably be higher than $10 \mathrm{mg}$ (f.i. $20 \mathrm{mg}$ ), but this has to be confirmed in pharmacodynamic studies. Considering the results found in this study, the micro-enema appears to be the optimal rectal formulation for temazepam with a short $t_{\max }$ and a $F_{\text {rel }}$ equivalent to 1. Taking into account the ease of self-administration and the ease of pharmaceutical (bulk-)preparation, the PEG-suppository appears to be a possible alternative formulation, if accepting its longer $t_{\max }$ and thus a slower therapeutic effect.

Temazepam from rectally administered liquid-filled capsules was overall unpredictably and inconsistently absorbed, despite good results in some individuals. Since oral absorption was fast and reproducible, it is therefore likely that the small volume of fluid in the rectum may be the limiting step in the absorption process of rectally administered capsules. Also, the intraluminal pressure may have a small influence on the release profiles of rectally administered drugs. Apparently, these factors were highly variabel within the tested population, resulting in an variable lagtime and bioavailability of temazepam in rectally administered liquid-filled capsules. Apart from the pharmacokinetic aspects, the difficulties in (self-) administration makes the liquid-filled capsule unsuitable for rectal use. The current practice of rectal administration of temazepam in a liquid-filled capsule should therefore be discouraged.

\section{Conclusion}

Based on pharmacokinetic data, rectal administration of temazepam can be effective, dependent on the formulation used.

Both the micro-enema, containing temazepam in a solution of ethanol, glycofurol and water, and the suppository with a polyethylene glycol base, can be used as rectal formulations of temazepam. Further pharmacodymanic studies are needed for determination of the proper dosage. The commercially available, liquid-filled capsule is less suitable for rectal administration.

\section{Acknowledgments}

The authors are very much indebted to all volunteers for their participation. The authors thank M. Grob and $\mathrm{P}$. Vrijlandt for their assistance in blood-sampling, T. Knuif for his assistance in analysing and Dr. K. Neef and $\mathrm{Dr}$. H-J. Guchelaar for their critically reading of the article. 


\section{References}

1 Bittencourt P, Richens A, Toseland PA, Wicks JFC, Latham AN. Pharmacokinetics of the hypnotic benzodiazepine temazepam. Br J of Clin Pharm 1979;8:37S-38S.

2 Fucella LM. Bioavailability of temazepam in soft gelatin capsulcs. Br J of Clin Pharm 1979;8:31 S-35S

3 Müller BW. Suppositorien. $1^{\text {st }}$ ed. Stuttgart: Wissenschaftliche Verlagsgesellschaft, 1986

4 Fucella LM. Study of the physiological availability of temazepam in man. Int.). of Clin.Pharm., Therapy and Toxicology. 1972; 64,303-309.

5 European Pharmacopoeia $2^{\text {nd }}$ Ed. Den Haag: Stadsuityeverij, 1993

6 Sande SA, Kralsen J. Curve fitting of dissolution data by personal computer. Int. J. Pharm. 1989; 55: 193-198

7 Uges DRA en medewerkers. Methods for the analysis of some xenobiotics in bodyfluids; Benzodiazepinen. Ziekenhuisfarmacie 1992;8:120-124

8 Vaart FJ van de. Guidelines for analytical validation. Ph Weekbl 1992; 46; 1229-1235

9 Schwarz HJ. Pharmacokinetics and metabolism of temaze. pam in man and several animal species. Br / Clin Pharmacol 1979; 8: 23S-29S.

10 Dhillon S, Richens A. Bioavailability of rectally-administered diazepam in adult epileptic patients. $\mathrm{Br} / \mathrm{Clin}$ Pharmacol $1981 ; 11: 437$.

11 Uges DRA. Referentiewaarden van klinisch-farmaceutische en toxicologische bepalingen (Therapeutic ranges of clinicalpharmaceutical and toxicological assays). Medicatiebegeleiding $1^{\text {st }}$ ed. Houten: Bohn Stafleu van Loghum, 1990; 421449. Ed.: de Smet PAGM, van Loenen $A C$, Offerhaus $L$, van der Does $\mathrm{E}$.

AIDS Clinical Trials. Guidelines for Design and Analysis. Edited by Dianne M. Finkelstein and David A. Schoenfeld. Published by Wiley-Liss, Inc., New York, 1995.

ISBN 0-471-58823-7, hard cover. 458 pp., $£ 41$,-

"AIDS Clinical Trials" is intended for use by clinicians and statisticians in order to gain more insight into the design, conduct and interpretation of clinical trials in populations infected with HIV. The book does not attempt to provide a background for the basic principles of clinical trials; specific aspects involving clinical trials in the HIV-infected population are highlighted. AIDS is different from other life-threatening diseases for which treatments are routinely studied in clinical trials. Infection with HIV is a chronic illness, making a long follow-up period necessary to detect improvement of for instance survival. However, current clinical practice is changing rapidly according as our knowledge of the pathogenesis of the virus is increasing. New antiretroviral drugs, drugs to treat opportunistic infections or HIV-related malignancies become available to scientists. These scientists are confronted with a generally well informed population that demands right of say in the design of clinical trials and puts pressure upon the access of new drugs. These issues generate intense and often conflicting concerns for scientists designing clinical trials to evaluate AIDS therapies.

The book has been divided in five parts. The first part gives an overview of the history and course of HIV-infection and the design of traditional trials and trials regarding the accelerated approval program of the FDA.

In the second and most voluminous part, the design of the study protocol, aspects regarding quality of life, selection of endpoints, and the use of $\mathrm{CD}_{4}{ }^{+}$ lymphocyte count as a measure of efficacy are discussed. Furthermore, statistical issues like sample size calculations, interim monitoring and the analysis of data from trials with combinations of drugs are reviewed. An excellent chapter regarding the statistical background of equivalence trials (a design often used in this area) is provided. The third part gives insight into specific problems encountered in trials in HIV-infected women and children. In the fourth part prophylactic HIV vaccine trials and large simple trials of HIV therapies are considered. The book is completed with a description of trials with an economic or epidemiological objective.

Altogether, a broad overview is presented of the design, conduct and analysis of clinical trials in HIV infected individuals. Both clinicians and statisticians will find interesting knowledge of statistics and medical practice, respectively; however, they will not find much new information regarding their own profession.

In general, literature references are up to date. Some aspects are described repeatedly in several chapters. Furthermore, regarding the registration of antiretroviral drugs, the book is aimed at the United States of America. Any reference to registration authorities other than the FDA is lacking.

"AIDS Clinical Trials" can be recommended to clinicians interested in statistics and statisticians who need clinical background information. Besides, the book will provide useful information to anyone interested in clinical trials in the HIV infected population.
Richard M.W. Hoetelmans. AlDS Clinical Trials. Cuidelines for Design and Anatysis. Pharm Wortd Sci 1996,18(3): 119 01996 Nlumer Academic Publishers. Printed in the Netherlands.

Richard M.W, Hotetmans: Pharm D, Department of Pharmacy, Slotervaart Hospital, Louwesweg 6, 1066 EC Amsterdam, The Netherlands.

Accepted April 1996. 